\title{
Solitons on intersecting 3-branes
}

\section{William Cottrell, Akikazu Hashimoto and Mohandas Pillai}

Department of Physics, University of Wisconsin, Madison, WI 53706, U.S.A.

E-mail: wcottrell@wisc.edu, aki@physics.wisc.edu, mpillai@wisc.edu

ABSTRACT: We consider a system consisting of a pair of D3 branes intersecting each other along a line such that half of the 16 supersymmetries are preserved. We then study the existence of magnetic monopole solutions corresponding to a D1-brane suspended between these D3 branes. We consider this problem in the zero slope limit where the tilt of the D3-branes is encoded in the uniform gradient of the adjoint scalar field. Such a system is closely related to the non-abelian flux background considered originally by van Baal. We provide three arguments supporting the existence of a single magnetic monopole solution. We also comment on the relation between our construction and a recent work by Mintun, Polchinski, and Sun.

KEYwords: Brane Dynamics in Gauge Theories, D-branes

ARXIV EPRINT: 1406.5872 


\section{Contents}

1 Introduction 1

2 BPS equations, magnetic charges, and the energy bound 5

3 Magnetic monopole in an intersecting brane background $\quad 7$

$\begin{array}{ll}3.1 & \text { Existence of solution to the generalized Nahm equation }\end{array}$

3.2 Existence of regular field configuration with correct magnetic charge and asymptotics

3.3 BPS solution at linear order in $a \quad 9$

4 Conclusions $\quad 12$

\section{Introduction}

The Atiyah-Drinfeld-Hitchin-Manin (ADHM) construction [1] and the Nahm [2] construction are powerful techniques for generating soliton solutions in gauge field theories. Roughly speaking, these constructions work by relating a BPS condition for a localized object in $d$ transverse dimensions to a related BPS condition for a localized object in $4-d$ transverse dimensions through a reciprocity relation [3]. In the case of instantons $(d=4)$ and monopoles $(d=3)$ for gauge field theories in four dimensions, the reciprocal objects live in 0 or 1 transverse dimensions. The reciprocal data, known respectively as the ADHM and Nahm data, are easier to assemble. Once they are assembled, they can be transformed to construct the instanton and monopole solutions systematically. Unlike the method based on an ansatz, the ADHM and Nahm constructions can formally construct multi soliton solutions and provide a framework to study issues such as the moduli spaces associated with these solitons.

BPS instantons and monopoles have natural interpretations in the context of world volume gauge theories on D-branes [4]. Instantons correspond to $\mathrm{D} p-\mathrm{D}(p+4)$ brane bound states, and BPS monopoles correspond to $\mathrm{D} p$-branes suspended between a pair of $\mathrm{D}(p+2)$ branes. The ADHM and the Nahm constructions themselves have string theory interpretations $[5,6]$. These relationships between BPS solitons and string theory are well known and are reviewed, e.g., in $[7,8]$.

The simple Prasad-Sommerfield monopole [9] on the world volume of a stack of D3branes preserves half of the 16 supersymmetries of the $\mathcal{N}=4$ supersymmetric Yang Mills theory living on its world volume. The reciprocal Nahm equation can also be viewed as a codimension one configuration of $\mathcal{N}=4$ supersymmetric Yang-Mills theory preserving half of the 16 supersymmetries [10]. This is not very surprising in light of the fact that in reciprocity, one is basically looking at the same system from a slightly different point 
of view. When a D1-brane is suspended between a pair of D3-branes, the D1-D3 system combine to form a funnel-like structure [11]. The monopole solution corresponds to looking at the system from the D3-brane point of view, and the Nahm equation corresponds to looking at the system from the D1-brane point of view.

Let us imagine the BPS monopole being described in terms of D3 and D1 branes oriented as follows:

\begin{tabular}{c|cccccccccc} 
& 0 & 1 & 2 & 3 & 4 & 5 & 6 & 7 & 8 & 9 \\
\hline D1 & $\circ$ & & & & & & $\circ$ & & & \\
D3 & $\circ$ & $\circ$ & $\circ$ & $\circ$ & & & & & &
\end{tabular}

The Nahm equation effectively describes the 1/2 BPS configuration of a D1 world volume embedded transversely in $X_{1}, X_{2}$, and $X_{3}$ direction, as well as the Wilson line $A_{6}$.

Recently, one of us constructed the generalization to Nahm equation where the number of supersymmetries preserved was reduced from $1 / 2$ to $1 / 4$ [12]. The generalized Nahm equation involves five set of scalars, and can be viewed as the world volume theory on D1-brane in the presence of D3 branes oriented as follows:

\begin{tabular}{c|cccccccccc} 
& 0 & 1 & 2 & 3 & 4 & 5 & 6 & 7 & 8 & 9 \\
\hline D1 & $\circ$ & & & & & & $\circ$ & & & \\
D3 & $\circ$ & $\circ$ & $\circ$ & $\circ$ & & & & & & \\
D3 $^{\prime}$ & $\circ$ & & & $\circ$ & $\circ$ & $\circ$ & & & &
\end{tabular}

Aspects of the dynamics of the fundamental strings in such a configuration was discussed in [13]. With regards to the magnetic monopoles, the Nahm equations should describe the embedding of the D1 brane world volume into $X_{1}, X_{2}, X_{3}, X_{4}$, and $X_{5}$ transverse coordinates as well as the Wilson line $A_{6}$. Concretely, the generalized Nahm equation, in terms of complex combinations

$$
\begin{aligned}
\mathcal{X} & \equiv X^{1}+i X^{2} \\
\mathcal{Y} & \equiv X^{4}+i X^{5}, \\
\mathcal{A} & \equiv A_{6}+i X^{3}
\end{aligned}
$$

consists of the following complex equations,

$$
\begin{aligned}
\frac{\mathcal{D} \mathcal{X}}{\mathcal{D} y} & =0, \\
\frac{\mathcal{D} \mathcal{Y}}{\mathcal{D} y} & =0, \\
{[\mathcal{X}, \mathcal{Y}] } & =0,
\end{aligned}
$$

and one real equation,

$$
\frac{d}{d y}(\mathcal{A}-\overline{\mathcal{A}})-[\mathcal{A}, \overline{\mathcal{A}}]+[\mathcal{X}, \overline{\mathcal{X}}]+[\mathcal{Y}, \overline{\mathcal{Y}}]=0,
$$

where $y$ is the world volume coordinate along $X_{6}$. When restricted to the case where $\mathcal{Y}=0$, this set of equations reduces to the standard Nahm equations. Just as in the standard Nahm 
construction, the presence of D3 and D3 ${ }^{\prime}$ branes is encoded in the boundary and junction condition at appropriate points along $y$, as was also discussed in [12].

The simplest, although somewhat trivial, solution of the generalized Nahm system one could consider is to place a single D1-brane suspended between a D3-brane at $y=0$ and a D3'-brane at $y=L$. The world volume theory on the D1 will be abelian, with $\mathcal{X}$ and $\mathcal{Y}$ fixed at the respective positions of the D3' and the D3 branes along these coordinates. One can also work in a gauge where $\mathcal{A}$ is a constant and parameterizes the one complex dimensional branch of the moduli space.

Perhaps the second simplest solution one could imagine is the case where there are two D1-branes suspended between the same D3 and D3' branes, free to move independently along $\mathcal{A}$. Such a solution would require boundary conditions with poles at the positions of D3 and D3' branes. It turns out, however, that such a solution to the Nahm equation does not exist as was shown in [12]. This non-existence claim is consistent with the expectation that in the S-dual picture, corresponding to two D3 branes suspended between an NS5 brane and an NS5' brane, an instanton effect gives rise to a repulsive force between the D3-branes preventing them from finding a stationary state. The basic physics behind this phenomenon can be traced back to the instanton generated superpotentials in $3 d \mathcal{N}=2$ Yang-Mills theory to which this system flows [14]. It should be emphasized that this instanton effect gets manifested classically when formulated in terms of the generalized Nahm equation. This exchange of classical and quantum phenomenon happens as a consequence of S-duality.

Even if a simple solution to the generalized Nahm equation for multi-monopole configurations does not exist, the solution corresponding to the singe monopole case is perfectly sensible. It is then natural to contemplate what the soliton configuration corresponding to these Nahm data by reciprocity could be. There are however some challenges in pursuing this simple query. First, the concept of reciprocity where one solves the construction equation and derives the soliton solution is not developed for the generalized Nahm equations. Second, it is not obvious how to chose the world volume between the D3 and the D3' brane on which to construct the soliton. One can in fact view the system as $\mathcal{N}=4 \mathrm{SYM}$ living on both D3 and D3' which interact through their intersection, on which some localized $33^{\prime}$ states live. From this point of view, the soliton we are after appears to take the form of a kink along the interface. The nature of such a kink solution has been a mystery. This issue was re-visited recently in [15] where it was argued that the kinetic term for the hypermultiplets at the interface must take on a non-canonical form to respect certain periodicity conditions expected in the field space. It was also argued that the interface theory is not strictly decoupled as a field theoretic system.

In this article, we will take a slightly different approach to the problem of identifying the soliton on the 1/4 BPS intersection of D3-branes. Let us imagine the 1/2 BPS monopole configuration as is illustrated in figure 1(a), with the D3 branes extended along the $x_{1}$ and $x_{2}$ directions. The $1 / 4$ BPS configuration considered in [15] is illustrated in figure $1(\mathrm{~b})$ where the $\mathrm{D} 3^{\prime}$ is now extended along the $x_{4}$ and $x_{5}$ directions. What one can now do to the configuration in figure $1(\mathrm{~b})$ is to rotate it in the $\left(x_{1}, x_{4}\right)$ plane and the $\left(x_{2}, x_{5}\right)$ to make it look like the configuration illustrated in figure 1(c). 


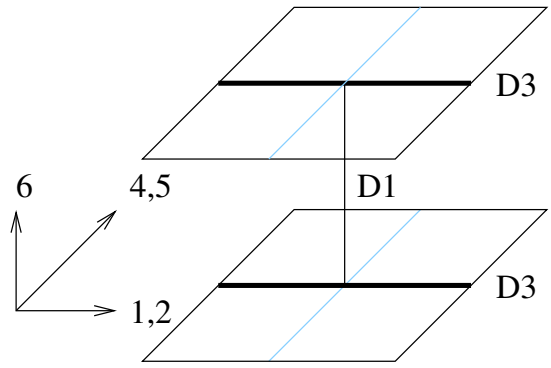

(a)

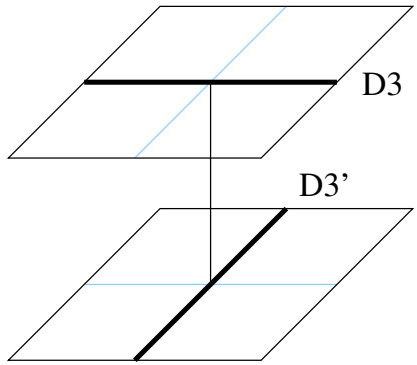

(b)

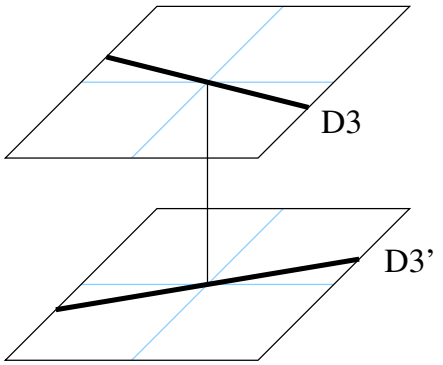

(c)

Figure 1. The brane configurations describing (a) ordinary BPS monopole, (b) monopole on intersecting D3 system, and (c) a monopole on an intersecting brane rotated by some angle. The configuration (b) requires considering U(1) fields on D3 and D3' as well as some degrees of freedom at the intersection. The configuration (c) can be understood as a configuration in $\mathrm{U}(2)$ field theory with linear field background (1.9).

In the absence of the monopole, the configuration of D3-brane illustrated in figure 1(c) was considered in [16]. It can be viewed as a T-dual of the non-abelian flux configuration considered originally by Van Baal in [17]. Using the field variables

$$
\Phi_{1}=\frac{1}{2 \pi \alpha^{\prime}} X_{4}, \quad \Phi_{2}=\frac{1}{2 \pi \alpha^{\prime}} X_{5}, \quad \Phi_{3}=\frac{1}{2 \pi \alpha^{\prime}} X_{6}
$$

the background field corresponding to the brane configuration illustrated in figure 1(c) can be expressed in the form

$$
\Phi_{1}=a x_{1} \sigma_{3}, \quad \Phi_{2}=-a x_{2} \sigma_{3}, \quad \Phi_{3}=v \sigma_{3}
$$

where $v$ has the dimension of mass and $a$ has the dimension $m^{2}$. The angle between the two D3 branes say in the $\left(x_{1}, x_{4}\right)$ plane is parameterized as

$$
\tan (\theta / 2)=2 \pi \alpha^{\prime} \times a .
$$

Such a configuration has a clean field theory decoupling limit $\alpha^{\prime} \rightarrow 0$ as a $\mathcal{N}=4$ theory with gauge group $\mathrm{U}(2)$. The background breaks half of the 16 supersymmetries. The $\mathrm{W}-$ bosons corresponding to the zero slope limit of the $33^{\prime}$-strings gives rise to a tower of states with masses of order $m^{2} \sim a[16,17]$, with a massless state at the bottom of the tower.

We are now ready to formulate the problem we wish to solve: what is the field configuration corresponding to adding a single Prasad-Sommerfield monopole in the background (1.9)? Such a soliton is expected to preserve $1 / 4$ of the supersymmetries and is the natural object to identify as the monopole associated to the simple solution of the generalized Nahm equation. We were unable to construct the exact solution for this object. However, we are able to offer several arguments supporting the existence of such a solution.

This article is organized as follows. We begin by setting up the field equations in section 2. Since the soliton we expect to find is supersymmetric, we will describe the relevant BPS equations instead of the Euler Lagrange equations. We will also recall the relevant 
formulas for defining the magnetic charge and the energy bounds implied by supersymmetry. In section 3, we will present our arguments supporting the existence of this soliton solution. We conclude in section 4 by discussing the how our findings relates to [15].

\section{BPS equations, magnetic charges, and the energy bound}

In this section, we construct the field equations expected to support the soliton solution which we outlined in the introduction. The physical system is basically $\mathcal{N}=4$ SYM in $3+1$ dimensions, which is well known to be a dimensional reduction of $\mathcal{N}=1 \mathrm{SYM}$ in $9+1$ dimensions. The solution we are seeking is static. In order to accommodate the magnetic charge and the background (1.9), we need to allow the fields $A_{1}, A_{2}, A_{3}, \Phi_{1}, \Phi_{2}$, and $\Phi_{3}$ to take on non-trivial values. One can embed that into six dimensional Yang-Mills theory dimensionally reduced to three.

The BPS equation can be inferred by imposing the standard gaugino condition

$$
\not F \chi=0
$$

for $\chi$ further constrained by

$$
\chi=\Gamma^{1236} \chi=\Gamma^{1245} \chi
$$

The first projection encodes the supersymmetry expected to be preserved by the monopole and the second projection encodes the supersymmetry preserved by the background (1.9). The BPS field equation inferred from these constraints have been worked out in $[18,19]$ and we can simply read them off as follows:

$$
\begin{aligned}
& F_{34}=-F_{65} \\
& F_{35}=F_{64} \\
& F_{15}=F_{24} \\
& F_{14}=-F_{25} \\
& F_{16}=F_{32} \\
& F_{26}=-F_{31} \\
& F_{36}=F_{45}-F_{12}
\end{aligned}
$$

In terms of the gauge fields and the adjoint scalar fields, these equations become

$$
\begin{aligned}
& D_{1} \Phi_{2}=D_{2} \Phi_{1} \\
& D_{1} \Phi_{1}=-D_{2} \Phi_{2} \\
& D_{3} \Phi_{1}=-i e\left[\Phi_{2}, \Phi_{3}\right] \\
& D_{3} \Phi_{2}=-i e\left[\Phi_{3}, \Phi_{1}\right] \\
& D_{1} \Phi_{3}=-F_{23} \\
& D_{2} \Phi_{3}=F_{13} \\
& D_{3} \Phi_{3}=-F_{12}-i e\left[\Phi_{1}, \Phi_{2}\right] .
\end{aligned}
$$


We are working in the physics convention where

$$
D_{i} \Phi \equiv \partial_{i} \Phi-i e\left[A_{i}, \Phi\right] .
$$

These equations can further simplified by noting that in terms of complex combinations

$$
\begin{aligned}
A & =A_{1}+i A_{2} \\
H & =A_{3}+i \Phi_{3} \\
\Phi & =\Phi_{1}-i \Phi_{2} \\
\mathcal{D} & =D_{1}+i D_{2} \\
\mathcal{D}_{3} & =\partial_{3}-i e[H, \cdot]
\end{aligned}
$$

the equations become 3 complex equations

$$
\begin{aligned}
\mathcal{D} \Phi & =0 \\
\mathcal{D}_{3} \Phi & =0 \\
\mathcal{D} H-\mathcal{D}_{3} A & =i e[H, A]
\end{aligned}
$$

and one real one

$$
\partial_{3}(H-\bar{H})=-(\mathcal{D} A-\overline{\mathcal{D}} \bar{A})-i e[H, \bar{H}]-i e[A, \bar{A}]-i e[\Phi, \bar{\Phi}] .
$$

These equations are remarkably similar to the complex gauge formalism used in [12] although here we are describing the space-time BPS equations instead of the Nahm equations.

These fields respect the energy bound [19] which can be written in a topological form

$$
\begin{aligned}
\frac{1}{4} \operatorname{Tr} F_{a b}^{2} & =\frac{1}{8} \operatorname{Tr}\left(F_{a b}+\frac{1}{4} \epsilon_{a b c d e f} T_{c d} T_{e f}+\kappa T_{a b} T_{c d} F_{c d}\right)^{2}-\frac{1}{16} \epsilon_{a b c d e f} T_{a b} \operatorname{Tr} F_{c d} F_{e f} \\
& >-\frac{1}{16} \epsilon_{a b c d e f} T_{a b} \operatorname{Tr} F_{c d} F_{e f} \\
& =-\frac{1}{4} \epsilon_{a b c d e f} T_{a b} \partial_{c} \operatorname{tr}\left(A_{d} \partial_{e} A_{f}-i \frac{2}{3} A_{d} A_{e} A_{f}\right)
\end{aligned}
$$

where in our convention, $T_{36}=-1, T_{12}=-1$, and $T_{45}=1$.

Let us subject these equations to some tests. The background (1.9) satisfies all the BPS equations. The only non-trivial equation is

$$
\mathcal{D} \Phi=\left(\partial_{1}+i \partial_{2}\right)\left(i a\left(x_{1}+i x_{2}\right) \sigma_{3}\right)=0 .
$$

The energy of this BPS state comes from

$$
\int d^{4} x T_{36} F_{24} F_{15} \sim \int d^{4} x a^{2}
$$

which diverges due to the infinite volume factor. This is what one expects from having a finite energy density.

Another quick test is the energy of ordinary Prasad-Sommerfield monopole which is finite and comes from the term

$$
-\frac{1}{8} \epsilon_{45 c d e f} T_{45} \operatorname{Tr} F_{c d} F_{e f}
$$

which reduces to the standard expression for the magnetic charge. 


\section{Magnetic monopole in an intersecting brane background}

Now that we have assembled our field equations (2.23)-(2.26) and tested it for the case of background (1.9) and the case of ordinary Prasad-Sommerfield background, let us turn to the exercise of combining these ingredients. What we wish to find is a soliton solution corresponding to placing a BPS magnetic monopole in the background which is asymptotically (1.9).

Strictly speaking, this problem should be cast in the context of finding a regular solution to (2.23)-(2.26) with the appropriate boundary condition at infinity. So far, we have been unsuccessful at presenting such a solution, mainly due to the lack of enough symmetries and likely lack of imagination. Instead of presenting explicit solutions, we will present three arguments which strongly suggests that such a solution must exist. The three arguments are

1. That a solution to the generalized Nahm equation exists

2. That a solution in the topological sector we seek exists and should relax to the BPS solution upon cooling, and

3. That a solution we seek can be found to linear order in $a$.

These do not constitute a proof, but are nonetheless quite suggestive. We will further comment on the plausibility of our arguments in section 4 .

\subsection{Existence of solution to the generalized Nahm equation}

One argument, which we alluded to in the introduction, is the fact that a solution exists for the generalized Nahm equations (1.4)-(1.7) corresponding to a single monopole arising from D1-branes stretched between tilted D3 and D3' branes. In the case of a single D3, the generalized Nahm equation and the boundary condition is somewhat trivial [12]. The solution is a constant and does not rely too sensitively on the orientation of the D3 branes. As such, this may be seen as a rather weak argument. Nonetheless, this solution does have the expected moduli space. Also, the Nahm analysis is sophisticated enough to know that multi-monopole configuration does not exist as a static, supersymmetric state. Presumably, there is a suitable generalization of the Nahm's procedure for constructing the monopole solution for the single monopole case, which will settle all these issues. But in the absence of that, we can take the existence of the solution to the generalized Nahm equation as a circumstantial evidence that a corresponding monopole exists.

\subsection{Existence of regular field configuration with correct magnetic charge and asymptotics}

As a second argument supporting the existence of the magnetic soliton solution in background (1.9), we observe that it is straightforward to construct a field configuration which does not solve the equation of motion but nonetheless has the correct asymptotics and the charges. The configuration satisfies, but does not saturate, the BPS energy bound (2.27). The energy of the configuration, relative to (1.9), is finite and is slightly higher than the 
expected energy of the monopole. It would then stand to reason that by starting with this configuration and letting the system relax to the lowest energy state in the charge sector, one would achieve the soliton state we are after.

The field configuration we have in mind is extremely simple. We start with the standard Prasad Sommerfield solution in the string gauge, where the scalar field is diagonal. Explicitly,

$$
\begin{aligned}
\mathcal{A}_{\mu} & =-\epsilon_{i j 3} \frac{\hat{r}}{e r} \frac{1}{1+\cos \theta} \\
W_{i} & =\frac{u(r)}{e r} v_{i} \\
\varphi & =h(r)
\end{aligned}
$$

where in the notation of [8],

$$
\mathcal{A}_{\mu}=A_{\mu}^{(3)}, \quad W_{\mu}^{ \pm}=\frac{1}{\sqrt{2}}\left(A_{\mu}^{(1)} \pm i A_{\mu}^{(2)}\right), \quad \varphi=\Phi_{3}^{(3)} .
$$

The index in parenthesis is the $\mathrm{SU}(2)$ adjoint index, $v_{i}$ encode angular dependence,

$$
\begin{aligned}
& v_{1}=-\frac{i}{\sqrt{2}}\left[1-e^{i \phi} \cos \phi(1-\cos \theta)\right] \\
& v_{2}=\frac{1}{\sqrt{2}}\left[1+i e^{i \phi} \sin \phi(1-\cos \theta)\right] \\
& v_{3}=\frac{i}{\sqrt{2}} e^{i \phi} \sin \theta
\end{aligned}
$$

and

$$
\begin{aligned}
u(r) & =\frac{e v r}{\sinh (e v r)} \\
h(r) & =v \operatorname{coth}(e v r)-\frac{1}{e r} .
\end{aligned}
$$

For the Prasad-Sommerfield soliton, we are also setting

$$
\Phi_{1}=\Phi_{2}=0
$$

Let us now consider generalizing this solution by turning on, by hand, the fields

$$
\Phi_{1}=a x_{1} \sigma_{3}, \quad \Phi_{2}=-a x_{2} \sigma_{3}
$$

which is precisely the form of the background (1.9).

This will not be a solution to the Yang-Mills equation of motion. Nonetheless, it is a field configuration for which

1. at large radius far away from the monopole, the background asymptotes exactly to (1.9), and 
2. because (1.9) vanishes near the origin and the Prasad-Sommerfield monopole is a regular solution aside from Dirac string singularity and has finite energy, the composite configuration will also be regular up to a Dirac string.

The topological charge given by (2.30) is unaffected by turning on the non-trivial background (1.9). As such, this solution is in the topological sector of the single magnetic monopole in the background (1.9).

One can also compute the energy of this field configuration. Explicitly evaluating the energy

$$
E=\int d^{3} x \operatorname{tr}\left[\frac{1}{2} F^{2}+D_{i} \Phi_{j} D_{i} \Phi_{j}+e^{2}\left(\left[\Phi_{i}, \Phi_{j}\right]\right)^{2}\right]
$$

will lead to a similar divergence as the one encountered in (2.29). However, what one should compute is the energy relative to the background (1.9) and that turns out to be finite. The computation of this relative energy takes the form

$$
\begin{aligned}
E=\int d^{3} x[ & \frac{e^{3} r^{3} v^{2} \operatorname{csch}^{2}(e r v)\left(2 e r v^{2}+3 e r v^{2} \operatorname{csch}^{2}(e r v)-4 v \operatorname{coth}(e r v)\right)+1}{e^{2} r^{4}} \\
& \left.+4 a^{2} e^{2} r^{2} v^{2} \sin ^{2}(\theta) \operatorname{csch}^{2}(e r v)\right] \\
= & \frac{4 \pi v}{e}+\frac{16 a^{2} \pi^{5}}{45 e^{3} v^{3}}
\end{aligned}
$$

The term proportional to $a^{2}$ is the positive definite term on the first line of (2.27) indicating the violation of the BPS condition. The term $4 \pi v / e$ is the contribution from the $(2.30)$ component of the topological term. The divergent contribution from (2.29) canceled in the computation of the relative energy.

The fact that there exists a field configuration which is finite in energy relative to the expected BPS bound, has the appropriate magnetic charge, and has the appropriate asymptotics strongly suggests that there exists a configuration which minimizes the energy in this sector of fields. This is a reasonably compelling argument supporting the existence of the soliton that we are after.

\subsection{BPS solution at linear order in $a$}

Finally, let us present a more quantitative analysis supporting the existence of the soliton solution under consideration. In this subsection, we describe the result of analyzing the BPS equation working only to linear order in the background parameter $a$ in (1.9). Of course, working to all orders in $a$ would constitute constructing the soliton entirely. Working to linear order in $a$ is a small step in that direction. However, as we will describe below, there are non-trivial tests that the system passes even at this somewhat crude level of analysis.

Since our goal is to study the response to the standard Prasad-Sommerfield solution by turning on the tilt parameter $a$ in (1.9) to first order in $a$, one can see from the form of (2.23)-(2.26) that it is consistent to assume only the complex field $\Phi$ is being modified. At this order, the equations for $H$ and $A$ in (2.25) and (2.26) are unaffected. So the problem reduces simply to that of solving (2.23) and (2.24) for $\Phi$ with the appropriate asymptotic behavior. 
In order to carry out this computation, we found it convenient to further transform the Prasad-Sommerfield solution in string gauge by a residual U(1) gauge symmetry

$$
A \rightarrow g^{-1} A g+i e g^{-1} \partial g
$$

for

$$
g=e^{-\frac{i}{2} \phi \sigma^{3}}
$$

This will bring the Prasad-Sommerfield background to take the form

$$
\begin{aligned}
& A=\frac{-i z}{r \rho}\left(u(\rho) \frac{\sigma^{1}}{2}+i u(r) \cos \theta \frac{\sigma^{2}}{2}-\frac{\cos \theta}{\sin \theta^{2}} \frac{\sigma^{3}}{2}\right) \\
& H=\frac{u(r)}{r} \sin \theta \frac{\sigma^{2}}{2}-i h(r) \frac{\sigma^{3}}{2} .
\end{aligned}
$$

Now, let us decompose $\Phi$ into its adjoint components

$$
\Phi=z \phi^{(a)}\left(z, x_{3}\right) \sigma^{a}
$$

Here, we have introduced a complex variable

$$
z=x_{1}+i x_{2}
$$

and

$$
\rho=|z|, \quad r^{2}=\rho^{2}+x_{3}^{2} .
$$

The fields $A$ and $\Phi$ appears to naturally have a unit of charge under global rotation in the $\left(x_{1}, x_{2}\right)$ plane. We have therefore parameterized $\Phi$ with a factor of $z$ pulled out explicitly, and will work with an ansatz that $\phi^{(a)}$ only depend on $\rho$.

$$
\Phi=z \phi^{(a)}\left(\rho, x_{3}\right) \sigma^{a} .
$$

Upon substituting this ansatz into (2.23) and (2.24), we obtain a system of coupled linear equations

$$
\begin{aligned}
\partial_{\rho}\left(\begin{array}{l}
\phi^{(1)}\left(\rho, x_{3}\right) \\
\phi^{(2)}\left(\rho, x_{3}\right) \\
\phi^{(3)}\left(\rho, x_{3}\right)
\end{array}\right) & =\left(\begin{array}{ccc}
0 & -\frac{i}{r} \frac{\cos \theta}{\sin \theta} \frac{u(r)}{r} \cos \theta \\
\frac{i}{r} \frac{\cos \theta}{\sin \theta} & 0 & -\frac{i}{r} u(r) \\
-\frac{u(r)}{r} \cos \theta & \frac{i}{r} u(r) & 0
\end{array}\right)\left(\begin{array}{l}
\phi^{(1)}\left(\rho, x_{3}\right) \\
\phi^{(2)}\left(\rho, x_{3}\right) \\
\phi^{(3)}\left(\rho, x_{3}\right)
\end{array}\right) \\
\frac{\partial}{\partial x_{3}}\left(\begin{array}{c}
\phi^{(1)}\left(\rho, x_{3}\right) \\
\phi^{(2)}\left(\rho, x_{3}\right) \\
\phi^{(3)}\left(\rho, x_{3}\right)
\end{array}\right) & =\left(\begin{array}{ccc}
0 & -i h(r)-\frac{u(r)}{r} \sin \theta \\
i h(r) & 0 & 0 \\
\frac{u(r)}{r} \sin \theta & 0 & 0
\end{array}\right)\left(\begin{array}{l}
\phi^{(1)}\left(\rho, x_{3}\right) \\
\phi^{(2)}\left(\rho, x_{3}\right) \\
\phi^{(3)}\left(\rho, x_{3}\right)
\end{array}\right) .
\end{aligned}
$$

We can attempt to solve these equations in two steps. First, restrict to $x_{3}=0$ for $(3.22)$ which then simplifies to

$$
\partial_{\rho}\left(\begin{array}{l}
\phi^{(1)}(\rho, 0) \\
\phi^{(2)}(\rho, 0) \\
\phi^{(3)}(\rho, 0)
\end{array}\right)=\left(\begin{array}{ccc}
0 & 0 & 0 \\
0 & 0 & -\frac{i}{r} u(r) \\
0 & \frac{i}{r} u(r) & 0
\end{array}\right)\left(\begin{array}{l}
\phi^{(1)}(\rho, 0) \\
\phi^{(2)}(\rho, 0) \\
\phi^{(3)}(\rho, 0)
\end{array}\right) .
$$


A general solution can be written

$$
\begin{aligned}
& \phi^{(1)}=C_{1} \\
& \phi^{(2)}=-i C_{2} \tanh (e v \rho / 2)-i C_{3} \operatorname{coth}(e v \rho / 2) \\
& \phi^{(3)}=C_{2} \tanh (e v \rho / 2)-C_{3} \operatorname{coth}(e v \rho / 2) .
\end{aligned}
$$

Since we want the solution to asymptote to the form of (1.9) for large $\rho$, we set $C_{1}=0$ and $C_{2}=-C_{3}=a / 2$ so that the solution is

$$
\begin{aligned}
\phi^{(1)}(\rho, 0) & =0 \\
\phi^{(2)}(\rho, 0) & =i \frac{a}{\sinh (e v \rho)} \\
\phi^{(3)}(\rho, 0) & =a \operatorname{coth}(e v \rho) .
\end{aligned}
$$

The next step is to solve the for $x_{3}$ dependence for each $\rho$ using (3.28)-(3.30) as the initial condition for the system of first order equations (3.23). Note that with the initial condition at $x_{3}=0$ prescribed for all $\rho$, this system of equation is completely determined and the solution is unique. In order to get the solution we seek, however, we require that the large $x_{3}$ asymptotics be compatible with (1.9) for all $\rho$. This amounts to subjecting our system of equations to infinitely many constraints. So finding a solution which satisfies all these requirements is rather non-trivial.

Yet, such solution indeed exists, and can be written rather compactly. It is

$$
\begin{aligned}
\phi^{(1)} & =-\frac{a x_{3}}{\rho \sinh (e v r)} \\
\phi^{(2)} & =i \frac{a r}{\rho \sinh (e v r)} \\
\phi^{(3)} & =a \operatorname{coth}(e v r) .
\end{aligned}
$$

This solution has the correct asymptotics for large $\rho$ as well as large $x_{3}$. The subleading corrections appear to be exponentially suppressed at large distances. The $\phi^{(i)}$ may appear to be diverging near the origin, but since we defined them relative to $\Phi^{(i)}$ with a factor of $z$ in (3.18), this is a perfectly regular field configuration. We have arrived at this solution mainly by trial and error, but the relative simplicity in the form of the solution suggests that there must be some hidden structure. We have identified one invariant, namely

$$
\frac{d}{d x_{3}}\left(\left(\phi^{(1)}\right)^{2}+\left(\phi^{(2)}\right)^{2}+\left(\phi^{(3)}\right)^{2}\right)=0 .
$$

Same is true about the derivative with respect to $\rho$. These follow essentially from the antisymmetry of $3 \times 3$ matrices in (3.22) and (3.23). Presumably there are few more invariants to allow these solutions to be derived in such compact forms, but we have been unsuccessful at identifying them.

In order to take this analysis to the next level, one should see if the BPS equations can be solved to order $\mathcal{O}\left(a^{2}\right)$ without distroying the asymptotic behavior at infinity and regularity near the origin. 


\section{Conclusions}

In this article we have constructed a field theory configuration corresponding to intersecting D3-branes breaking half of the supersymmetries, and argued for the existence of a soliton solution corresponding to a single magnetic monopole constrained to move along the intersection in this background. While we have not succeeded in finding an explicit form for the monopole, we provided three arguments in support of its existence.

The problem we consider is very similar to the problem considered by [15], so let us take a moment to discuss the relation between our analysis and that of [15]. The main difference in the setup can be summarized as the difference between the configurations illustrated in figure 1(b) and figure 1(c). The physical setup of [15] is the one illustrated in $1(\mathrm{~b})$, where the basic degrees of freedom are the 33 strings, $3^{\prime} 3^{\prime}$ strings, and the $33^{\prime}$ strings in the zero slope limit. In the zero slope limit, all of these states are in the lowest energy state. The $33^{\prime}$ lives in $1+1$ dimensions. In order to support the kink-like soliton corresponding to the D1-string, the authors of [15] were forced to consider a non-canonical form for the kinetic terms of the $33^{\prime}$ states. The dynamics was also described in terms of a renormalized effective field theory and the system resisted having a good UV completion which manifested in the form of singularity in the Kahler metric. The authors of [15] were forced to appeal to the full machinery of string theory to complete the dynamics dynamics.

The setup of $1(\mathrm{c})$, on the other hand, captures the intersection of D3 while staying inside the field theory framework. The $33^{\prime}$ states correspond to the off diagonal components of the adjoint gauge and matter fields. In the framework of tilted backgrounds, these $33^{\prime}$ states form a tower of states with masses of order $a$. By working at energies below the scale set by $a$, we can ignore all but the massless $33^{\prime}$ states, and in that limit, the degrees of freedom surviving in the dynamics are essentially the same as the setup of [15]. It appears then that states with masses of order $a$ are regulating the dynamics in the UV. It seems plausible then for the non-canonical kinetic term to arise from integrating out these massive $33^{\prime}$ states, and that a singularity arises when taking the limit $a \rightarrow \infty$. It would be interesting to understand this point better.

In this article, we focused primarily on the single monopole solution. Unlike in the case of ordinary BPS monopoles, we do not expect multi monopole solutions to be BPS in the background (1.9) with non-vanishing $a$. One way to show that a static multi-monopole configuration can not exist is to compute the force experienced by these states. The forces experienced by the kinks constructed by [15] was computed recently in [20] and perhaps a similar method can be applied. Another interesting way to approach this issue is to study the moduli space dynamics from the perspective of Nahm data.

Finally, let us conclude by recalling that one of our initial motivations was to generalize the reciprocity of ADHM and Nahm procedure in order to formulate a systematic way to construct the monopole solutions. So far, we have not succeeded in formulating such a procedure. There are some previous works on generalizing the ADHM construction to higher dimensions [21], and hopefully, an exact solution for the monopole solution considered in this article can be constructed along these lines. 


\section{Acknowledgments}

This work supported in part by the DOE grant DE-FG02-95ER40896 and by funds from University of Wisconsin. AH would like to thank Peter Ouyang and Masahito Yamazaki for collaboration on related work which inspired this project. AH also thanks Joe Polchinski for an interesting discussion.

Open Access. This article is distributed under the terms of the Creative Commons Attribution License (CC-BY 4.0), which permits any use, distribution and reproduction in any medium, provided the original author(s) and source are credited.

\section{References}

[1] M.F. Atiyah, N.J. Hitchin, V.G. Drinfeld and Y. Manin, Construction of instantons, Phys. Lett. A 65 (1978) 185 [INSPIRE].

[2] W. Nahm, A simple formalism for the BPS monopole, Phys. Lett. B 90 (1980) 413 [INSPIRE].

[3] E. Corrigan and P. Goddard, Construction of instanton and monopole solutions and reciprocity, Annals Phys. 154 (1984) 253 [INSPIRE].

[4] M.R. Douglas, Branes within branes, hep-th/9512077 [INSPIRE].

[5] E. Witten, $\sigma$-models and the ADHM construction of instantons, J. Geom. Phys. 15 (1995) 215 [hep-th/9410052] [INSPIRE].

[6] D.-E. Diaconescu, D-branes, monopoles and Nahm equations, Nucl. Phys. B 503 (1997) 220 [hep-th/9608163] [INSPIRE].

[7] D. Tong, TASI lectures on solitons: instantons, monopoles, vortices and kinks, hep-th/0509216 [INSPIRE].

[8] E.J. Weinberg and P. Yi, Magnetic monopole dynamics, supersymmetry and duality, Phys. Rept. 438 (2007) 65 [hep-th/0609055] [INSPIRE].

[9] M.K. Prasad and C.M. Sommerfield, An exact classical solution for the 't Hooft monopole and the Julia-Zee dyon, Phys. Rev. Lett. 35 (1975) 760 [INSPIRE].

[10] D. Gaiotto and E. Witten, Supersymmetric boundary conditions in $\mathcal{N}=4$ super Yang-Mills theory, J. Statist. Phys. 135 (2009) 789 [arXiv:0804.2902] [InSPIRE].

[11] A. Hashimoto, The shape of branes pulled by strings, Phys. Rev. D 57 (1998) 6441 [hep-th/9711097] [INSPIRE].

[12] A. Hashimoto, P. Ouyang and M. Yamazaki, Boundaries and defects of $\mathcal{N}=4$ SYM with 4 supercharges. Part I: Boundary/junction conditions, JHEP 10 (2014) 107 [arXiv: 1404.5527] [INSPIRE].

[13] J. Erdmenger, Z. Guralnik, R. Helling and I. Kirsch, A world volume perspective on the recombination of intersecting branes, JHEP 04 (2004) 064 [hep-th/0309043] [INSPIRE].

[14] I. Affleck, J.A. Harvey and E. Witten, Instantons and (super)symmetry breaking in (2+1)-dimensions, Nucl. Phys. B 206 (1982) 413 [INSPIRE].

[15] E. Mintun, J. Polchinski and S. Sun, The field theory of intersecting D3-branes, arXiv: 1402.6327 [INSPIRE]. 
[16] A. Hashimoto and W. Taylor, Fluctuation spectra of tilted and intersecting D-branes from the Born-Infeld action, Nucl. Phys. B 503 (1997) 193 [hep-th/9703217] [INSPIRE].

[17] P. van Baal, $\mathrm{SU}(N)$ Yang-Mills solutions with constant field strength on $T^{4}$, Commun. Math. Phys. 94 (1984) 397 [InSPIRE].

[18] E. Corrigan, C. Devchand, D.B. Fairlie and J. Nuyts, First order equations for gauge fields in spaces of dimension greater than four, Nucl. Phys. B 214 (1983) 452 [inSPIRE].

[19] D.-s. Bak, K.-M. Lee and J.-H. Park, BPS equations in six-dimensions and eight-dimensions, Phys. Rev. D 66 (2002) 025021 [hep-th/0204221] [INSPIRE].

[20] D. Dorigoni and D. Tong, Intersecting branes, domain walls and superpotentials in $3 d$ gauge theories, JHEP 08 (2014) 119 [arXiv:1405.5226] [INSPIRE].

[21] E. Corrigan, P. Goddard and A. Kent, Some comments on the ADHM construction in 4k-dimensions, Commun. Math. Phys. 100 (1985) 1 [INSPIRE]. 